\title{
Commentary \\ The utility of ductal lavage in breast cancer detection and risk assessment \\ Susan M Domchek
}

The Rena Rowan Breast Center, University of Pennsylvania, Philadelphia, Pennsylvania, USA

Correspondence: Susan M Domchek, MD, The Rena Rowan Breast Center, University of Pennsylvania, 14 Penn Tower, 3400 Spruce Street, Philadelphia, PA 19104-4283, USA. Tel: +1 215615 3360; fax: +1 215662 6060; e-mail: susan.domchek@uphs.upenn.edu

Received: 3 December 2001

Accepted: 16 January 2002

Published: 1 February 2002
Breast Cancer Res 2002, 4:51-53

(C) 2002 BioMed Central Ltd

(Print ISSN 1465-5411; Online ISSN 1465-542X)

\begin{abstract}
Ductal lavage (DL) permits noninvasive retrieval of epithelial cells from the breast. Clinical development of this technique has been fueled largely by its potential, as yet unproven, to improve detection of breast cancer and definition of individual risk for development of breast cancer. Early studies demonstrate the feasibility of performing this technique, provide data on cellular yield and findings, and demonstrate the ability to measure molecular markers in DL fluid. However, the sensitivity and specificity of DL for the detection of breast cancer remains unknown, as does the significance of atypia, particularly mild atypia, when found in DL fluid. Although DL appears safe and the device is approved by the US Food and Drug Administration, DL is still best utilized in the setting of clinical trials designed to resolve issues of sensitivity, specificity, and localization.
\end{abstract}

Keywords: breast cancer, detection, ductal lavage, risk assessment

\section{Introduction}

Attempts to improve early detection of breast cancer and to provide individualized breast cancer risk assessment include methods to examine cellular material from the apparently normal breast. Although mammographic screening is an effective tool for detecting breast cancer, it has inherent and well described limitations [1]. In addition, mathematical models such as the Gail Model have been developed to predict breast cancer risk [2], but such models are better at assessing population-based risk rather than individual risk. Tamoxifen decreases risk for development of breast cancer by $50 \%$ in women who are at increased risk for the disease [3]. Unfortunately, tamoxifen has rare but serious side effects; therefore, additional information to refine risk stratification would be useful.
Nipple aspirate fluid (NAF) can be obtained from nonlactating women by simple suction methods. Several groups have examined the cytology of NAF in attempts to improve detection of breast cancer and predict risk based on cellular findings [4-7]. Although abnormal cytology in NAF is predictive of subsequent development of breast cancer, with a relative risk of 2.5-5 at 12.5 years [5], cellular yield from this technique is low. Atypia seen in fine-needle aspiration (FNA) of the breast, in which multiple aspirations are performed and pooled for analysis, also is associated with an increased risk for breast cancer, with a relative risk of 5 at 45 months [8]. The relative risk seen with atypia detected either in NAF or in FNA is consistent with that observed for atypical ductal hyperplasia when detected in surgical specimens [9-13] and suggests that atypia 
as identified by these methods may help further define individual risk for breast cancer.

\section{Ductal lavage}

In an effort to improve upon NAF and FNA, a DL system has been devised. This method involves suction of the nipple in order to localize NAF-yielding duct(s). NAFproducing ducts can then be cannulated using a microcatheter (Pro*Duct Health Inc., Menlo Park, CA, USA) and lavaged with saline in order to retrieve duct epithelial cells for cytological analysis. NAF-producing ducts are targeted for logistical reasons (NAF allows for the localization of the ductal orifice and subsequent cannulation) and because data suggest that women who produce NAF are at increased risk for the development of breast cancer [5]. Two recent studies $[14,15]$ examined applications of DL, including the ability of DL versus NAF to detect abnormal breast epithelial cells and the ability to use molecular techniques such as methylation-sensitive polymerase chain reaction (MSP) in DL specimens to identify abnormal cells.

\section{Detection of abnormal cytology}

Dooley et al. [14] examined 507 women with an elevated risk for breast cancer (based on elevated Gail index, prior history of invasive or noninvasive breast cancer, or presence of a BRCA mutation), but with low-suspicion physical examinations and mammograms, by performing nipple aspiration followed by DL. NAF and DL samples were obtained from 82 and $75 \%$ of women, respectively. The $\mathrm{DL}$ specimens were much more likely to have adequate cellular material for cytologic diagnosis ( $78 \%$ versus $27 \%$ ) and had higher median epithelial cell yields $(13,500$ epithelial cells per duct versus 120 epithelial cells per breast). Abnormal cytology was detected in $24 \%$ of DL samples: $17 \%$ were mildly atypical, $6 \%$ were markedly atypical, and $0.5 \%$ were termed malignant. For NAF these percentages were 6,3 , and $0.5 \%$, respectively. The two women with malignant cytology on $\mathrm{DL}$ were the same two with malignant cytology on NAF. DL was reasonably well tolerated, with a median pain rating of 24 versus 8 for nipple aspiration (scale $0-100$ ). In addition to breast pain, breast engorgement, and ecchymoses, two possible cases of cellulitis were identified. Eleven patients with abnormal cytology had further diagnostic studies, including imaging, ductoscopy and surgery. Four were diagnosed with ductal carcinoma in situ (DCIS). Details regarding the corresponding $\mathrm{DL}$ cytology of those patients have not been reported, or has the corresponding follow up in the two women with malignant cytology.

\section{Molecular markers}

In addition to cytology, DL may permit analysis of molecular markers associated with breast carcinomas. In a report by Evron et al. [15], specimens (excised tumors, duct endoscopy fluid, or DL samples) were examined by MSP for Cyclin D2, RAR- $\beta$ and Twist genes. At least one of the three genes was methylated in $96 \%$ of surgically excised primary breast tumors and in $57 \%$ of DCIS lesions, but in no normal breast tissue samples, demonstrating high sensitivity and specificity of this technique. Thirty-seven patients with biopsy-proven breast cancer underwent duct endoscopy, with direct visualization of the ducts, and lavage; the specimens were evaluated using MSP. In the 20 women in whom residual invasive carcinoma was identified histologically at re-excision, 17 (85\%) also had at least one methylated marker present in the duct fluid. Of the 13 women with residual DCIS or atypical ductal hyperplasia identified histologically at re-excision, four (30\%) had a methylated marker. All four women with negative reexcisions also had negative MSP.

Using cytologic examination and MSP, Evron et al. [15] also examined DL fluid from 56 women who were part of the NAF versus DL study described above [14]. Six of those women had DL specimens classified by cytology as atypical with substantial changes, or as malignant. Four of these six cases had a least one positive marker by MSP (67\%). Two of these four women (with both abnormal cytology and positive MSP) were further evaluated and found to have DCIS. These data demonstrate the feasibility of detection of molecular markers in DL specimens and suggest that $\mathrm{DL}$ may have a role to play in early detection.

\section{Limitations}

Data from the studies described above $[14,15]$ demonstrate that $\mathrm{DL}$ is a feasible outpatient procedure by which cellular material can be retrieved. In a handful of cases, evaluation of abnormal DL cytology led to a diagnosis of DCIS that was not initially detected by physical examination or mammography. DL enables greater cellular yield than NAF, and leads to fewer pathologic diagnoses of 'insufficient cellular material'. In addition, DL detects significantly more atypia than does NAF. Although it is possible that atypia from $\mathrm{DL}$ connotes the same risk as atypia from NAF and FNA, this remains to be confirmed with additional studies. DL detected more cases of mild atypia than did NAF. Far fewer cases of either marked atypia or malignancy were found by either method, and the detection rates of the two methods appeared to vary less substantially than for the detection of mild atypia. Atypia found on NAF has been reported as predictive of future breast cancer risk [5], which suggests that NAF detects the atypia that matters', but follow-up studies are needed to improve our understanding of the significance of mild atypia as detected by DL. Therefore, it is currently unclear how findings from DL should be incorporated into risk assessment. If a woman has previously decided not to take tamoxifen for chemoprevention, should she be encouraged to do so on the basis of mild atypia from a $D L$ sample? Given the uncertainties, the use of DL findings to stratify patients for tamoxifen prevention should be done within the context of a clinical trial. 
Second, the sensitivity and specificity of DL for the detection of invasive carcinoma is unknown. A negative DL should not be reassuring to a woman at high risk. In the study that established NAF atypia as a risk factor [5], the majority of patients who eventually developed breast cancer did not have abnormal cytology. Whether DL adds to the sensitivity of NAF is unknown. Data do not currently exist on the rate of abnormal cytology in non-NAF-yielding ducts. Thus, a normal lavage should not discourage a woman from routine screening or from taking tamoxifen for prevention if she is otherwise a candidate.

So what does a positive DL result mean and how should it be evaluated? Any algorithm will necessarily be based on extrapolation from NAF, given the limited follow-up data for DL. A practicing oncologist might view the issue as follows; should a woman go to mastectomy if DL repeatedly shows markedly atypical cells and all imaging studies are negative? Given the available data, this seems extreme. Follow-up data from published studies and further investigation are needed before patients can be meaningfully informed about the implications of $\mathrm{DL}$ results. Once clarified, DL may in time help to avoid unnecessary surgery and yet detect silent tumors.

\section{Conclusion}

DL remains a promising technique for early detection of breast cancer and for detection of premalignant lesions in high-risk women. Nonetheless, data regarding the sensitivity and specificity of $\mathrm{DL}$ in detecting breast cancer, its usefulness in risk stratification, and the significance of mild atypia as detected by $\mathrm{DL}$ are limited. The ability to perform polymerase chain reaction amplification on ductal epithelial cells retrieved by DL may allow for early detection of molecular markers that are associated with malignant transformation, such as promoter methylation changes or telomerase expression. The promise of DL is great, but many questions remain. Because of this, $\mathrm{DL}$ is best used in the context of clinical trials focused on breast cancer detection and prevention.

\section{Acknowledgement}

I thank Dr Barbara L Weber for helpful discussion.

\section{References}

1. Elmore JG, Barton MB, Moceri VM, Polk S, Arena PJ, Fletcher SW: Ten-year risk of false positive screening mammograms and clinical breast examinations. $N$ Engl J Med 1998, 338: 1089-1096.

2. Gail MH, Brinton LA, Byar DP, Corle DK, Green SB, Schairer C, Mulvihill JJ: Projecting individualized probabilities of developing breast cancer for white females who are being examined annually. J Natl Cancer Inst 1989, 81:1879-1886.

3. Fisher B, Costantino JP, Wickerham DL, Redmond CK, Kavanah M, Cronin WM, Vogel V, Robidoux A, Dimitrov N, Atkins J, Daly M, Wieand S, Tan-Chiu E, Ford L, Wolmark N: Tamoxifen for prevention of breast cancer: report of the National Surgical Adjuvant Breast and Bowel Project P-1 Study. J Natl Cancer Inst 1998, 90:1371-1388.

4. Sartorius OW, Smith HS, Morris P, Benedict D, Friesen L: Cytologic evaluation of breast fluid in the detection of breast disease. J Nat/ Cancer Inst 1977, 59:1073-1080.

5. Wrensch MR, Petrakis NL, King EB, Miike R, Mason L, Chew KL, Lee MM, Ernster VL, Hilton JF, Schweitzer R, et al.: Breast cancer incidence in women with abnormal cytology in nipple aspirates of breast fluid. Am J Epidemiol 1992, 135:130-141.

6. Sauter ER, Ross E, Daly M, Klein-Szanto A, Engstrom PF, Sorling $A$, Malick J, Ehya $\mathrm{H}$ : Nipple aspirate fluid: a promising noninvasive method to identify cellular markers of breast cancer risk. Br J Cancer 1997, 76:494-501.

7. Petrakis NL: Nipple aspirate fluid in epidemiologic studies of breast disease. Epidemiol Rev 1993, 15:188-195.

8. Fabian CJ, Kimler BF, Zalles CM, Klemp JR, Kamel S, Zeiger S, Mayo MS: Short-term breast cancer prediction by random periareolar fine-needle aspiration cytology and the Gail risk model. J Natl Cancer Inst 2000, 92:1217-1227.

9. Dupont WD, Page DL: Risk factors for breast cancer in women with proliferative breast disease. N Engl J Med 1985, 312:146151.

10. Dupont WD, Parl FF, Hartmann WH, Brinton LA, Winfield AC, Worrell JA, Schuyler PA, Plummer WD: Breast cancer risk associated with proliferative breast disease and atypical hyperplasia. Cancer 1993, 71:1258-1265.

11. Marshall LM, Hunter DJ, Connolly JL, Schnitt SJ, Byrne C, London SJ, Colditz GA: Risk of breast cancer associated with atypical hyperplasia of lobular and ductal types. Cancer Epidemiol Biomarkers Prev 1997, 6:297-301.

12. Palli D, Rosselli del Turco M, Simoncini R, Bianchi S: Benign breast disease and breast cancer: a case-control study in a cohort in Italy. Int J Cancer 1991, 47:703-706.

13. London SJ, Connolly JL, Schnitt SJ, Colditz GA: A prospective study of benign breast disease and the risk of breast cancer. JAMA 1992, 267:941-944.

14. Dooley WC, Ljung BM, Veronesi U, Cazzaniga M, Elledge RM, O'Shaughnessy JA, Kuerer HM, Hung DT, Khan SA, Phillips RF, Ganz PA, Euhus DM, Esserman LJ, Haffty BG, King BL, Kelley MC, Anderson MM, Schmit PJ, Clark RR, Kass FC, Anderson BO, Troyan SL, Arias RD, Quiring JN, Love SM, Page DL, King EB: Ductal lavage for detection of cellular atypia in women at high risk for breast cancer. J Natl Cancer Inst 2001, 93:1624-1632.

15. Evron E, Dooley WC, Umbricht CB, Rosenthal D, Sacchi N, Gabrielson E, Soito AB, Hung DT, Ljung B, Davidson NE, Sukumar S: Detection of breast cancer cells in ductal lavage fluid by methylation-specific PCR. Lancet 2001, 357:13351336. 